\title{
I PARADOSSI DI UN MENTITORE. GIACOMO CASANOVA TRA VERITÀ E SIMULAZIONE
}

\author{
Diego VARINI \\ Università di Parma
}

\begin{abstract}
En): This paper tries to outline the multiple plans and significances of simulation/dissimulation in Giacomo Casanova's Histoire de ma vie. Focusing on themes such as time perception and representation, the dialectic of dream and reality, the self-consciousness of the lying author and its earnest attitude and Casanova's political judgments, the "content of reality" and the revealing value of this bizarre autobiography emerge, even if concealed under the richness of implausible or plainly false accounts. Playing with elegance and irony the multiple instruments of the interplay between true and false, Casanova's autobiography stands as a forceful example of an experimental literary genre in which autobiography and novel share the same techniques and style: instead of a dialogue between the fictional and non-fictional plan, the Histoire de ma vie proposes the possibility of re-evaluating the fictional contents as the only possible faithful and tentatively "objective" narrative, while the lived reality is too often unable to represent itself out of the dissimulations of the subjectivity.
\end{abstract}

Keywords (En): Casanova ; Histoire de ma vie ; autobiography ; simulation ; eighteenth century

Parole-chiave (It): Casanova ; Histoire de ma vie ; autobiografia ; simulazione ; diciottesimo secolo

\section{Casanova, currenti calamo, alla ricerca del tempo perduto}

In un presente continuamente «teso a rifondare se stesso» (POULET, 1967 : 694), l'Histoire de ma vie offre una conferma inquietante alle osservazioni con le quali Paul Valéry (ragionando, nel 1927, dello stendhaliano Lucien Leuwen, in uno splendido intervento poi confluito nel primo volume di Varieté) annotava che «les auteurs de confessions ou de souvenirs ou de journaux intimes sont inévitablement les dupes de leur espoir de choquer; et nous, dupes de ces dupes» (VALÉRY, 1959 : 570-71). Ostaggi di questo ostaggio, è qualche volta legittimo concludere - come avviene, per esempio, ad un romanziere-critico della tempra di Philippe SOLLERS (1999 : 133) - che «Casanova si diverte della vita, la quale si diverte con lui». Eppure aveva colto nel segno, con profonda lucidità, un altro grande romanziere che di Casanova fu per decenni, nella prima metà del Novecento, un fervido cultore (non solo per ragioni di contiguità geografica): il veneto Giovanni Comisso, quando evocava con sgomento (in una pagina del 1958, posta a margine di una piccola scelta di sue traduzioni dall'Histoire de ma vie) quella memoria terribile che doveva avere perseguitato Casanova durante l'intera sua esistenza, «una facoltà vissuta e patita - osserva Ilaria CROTTI (2005 : 143) - come una sorta di condanna a strutturare e, nel contempo, a preservare dall'oblio il ricordo», all'insegna di «un peculiare tratto edonistico $[\ldots]$ dove piacere, appunto, memoria, dolore e scrittura si rispecchiano a vicenda». «Mi diverto perché non invento» confessa CASANOVA (1913: 71) in una celebre lettera (datata "Dux, 10 gennaio 
1791”) del carteggio con il suo corrispondente boemo Johannes Ferdinand Opiz (in un tempo nel quale la stesura dell'Historie de ma vie era pienamente ed energicamente avviata da almeno due anni); «scrivo tredici ore al giorno, che mi sembrano tredici minuti. Che piacere, ricordarsi dei propri piaceri. Ma che pena richiamarli in mente». Se fosse lecito prendere a prestito il proclama che RESTIF DE LA BRETONNE (1959 : XLI) fa campeggiare nell'introduzione al proprio Monsieur Nicolas: «ce n'est pas ma vie que je fais; c'est l'histoire d'un homme». Ma la frase, applicata a Casanova, assume subito una luce ambigua: perché il progetto di stendere il minuzioso ed esaustivo referto della propria vita, di tutta la propria vita nei quasi cinquant'anni «depuis 1725 jusqu'à l'an 1774», implica già in premessa «quella identificazione assoluta tra autore e opera che è il segno dei "grandi", almeno fino al Settecento e a Goethe» (ANGLANI, 1997 : 8). Per rifarsi alle parole di LEJEUNE (2004² : 20): «le paradoxe de l'autobiographie, c'est que l'autobiographie doit exécuter ce projet d'une impossible sincérité en se servant de tous les instruments habituels de la fiction». Lo sapeva bene Casanova, aldilà delle mille esibite professioni di scrupolosa fedeltà al «vero fattuale» che punteggiano il dettato dell' Histoire de ma vie: la prima e fondamentale "simulazione" che muove la macchina delle sue memorie si lega alla messa in opera di un poderoso artificio romanzesco, fondato su un procedimento di polifonica «teatralizzazione dell'autobiografia» (RUOZZI, $2012: 83$ ), per ricreare à rebours sulla carta, nella forzosa inazione del castello di Dux (ove trascorre, alle dipendenze del conte di Waldstein, una malinconica stagione senile), la pienezza ebbra di sensualità delle trascorse avventure giovanili. «Je faisais une expérience sur moi-même persuadé de ne pas me connaître assez bien. J'en étais curieux» (CASANOVA, $2013: 410$ ).

Un tale "esperimento" - mentre non contraddice i termini costitutivi del patto autobiografico teorizzato da Lejeune (fondato su una sostanziale identità fra il narratore e il personaggio che dice "io") - implica tuttavia un commercio sottile e continuo con gli artifici attraverso i quali la macchina retorica dell'affabulazione modifica i connotati del personaggio casanoviano al centro dell'autoritratto. In un lungo passaggio, di trasparente intonazione metapoetica, CASANOVA (2015a : 379) scrive per esempio:

Aucun peintre ne surpassa Raphaël dans la beauté des figures produites par son pinceau; mais si on avait demandé à Raphaël ce que c'était que cette beauté, dont il savait si bien les lois, il aurait répondu qu'il n'en savait rien, qu'il la savait par cœur, et qu'il croyait de l'avoir produite, quand il la voyait devant ses yeux. Cette figure me plait, devait il dire, elle est donc belle. Il devait remercier Dieu d'être né avec un excellent goût pour la beauté. Mais omne pulcrum difficile. [...] Observons combien un bon peintre est rare dans la classe de ceux qui se sont adonnés au talent de faire des portraits. C'est le genre le plus matériel de leur art. Il y en a de trois espèces. Ceux qui ressemblent, et enlaidissent: ils méritent selon moi d'être payés par des coups de bâtons, car ils sont impertinents, et ils ne conviennent jamais d'avoir fait la personne plus laide, ou moins belle. Le seconds, auxquels on ne peut pas refuser du mérite, sont ceux qui ressemblent parfaitement, et même à un point qui étonne, car la figure paraît parlante. Mais les rares, et très rares sont ceux qui ressemblent parfaitement, et en même temps ajoutent un caractère imperceptible de beauté à la figure qu'ils ont tracée sur les tableaux. Ces peintres sont dignes de la fortune qu'ils font.

Il discorso - modellato su un episodio insigne del classicismo rinascimentale (la pittura di Raffaello) - sembrerebbe in partenza lasciar supporre, anche da parte 
di Casanova, quella tipica aspirazione (agostiniana e petrarchesca) a «fissare in una immobilità esemplare l'esperienza irripetibile del vissuto, [che] costituisce uno dei caratteri più profondi e persistenti dell'Umanesimo» (VAROTTI, 1998 : 121). Ma le cose stanno in verità all'opposto, perché il fondamentale paradosso dell'operazione memorialistica orchestrata da Casanova è quello di non tendere ad alcuna esemplarità, di non ricomporsi all'insegna di nessun "fabula docet" o impianto moraleggiante, in un rifiuto radicale e insanabile dell'idea stessa di destino («une parole vide de sens», CASANOVA, 2015a : 125) che si traduce poi abissalmente in «connaissance fluente d'un objet fluent», se vogliamo dirla nei termini utilizzati da POULET (1977: 15) parlando una volta di Montaigne. ${ }^{1} \mathrm{E}$ in fondo la radice segretamente proustiana dell'Histoire de ma vie in quanto operamonstrum, elefantiaca e depistante opera-mondo indirizzata a negare se stessa, sulla scorta di una dialettica interminabile, fra sempre uguale e sempre altro, che funziona da ingrediente di inarrestabile dislocazione centrifuga, tendenzialmente in infinitum. Su questo aspetto restano illuminanti le parole di Giorgio FICARA (1999 : IX-X):

\begin{abstract}
Libro eccentrico anche tra i memoriali settecenteschi, [l'Histoire de ma vie] non ha altri obiettivi che restituire intatto il sapore del disordine e della felicità lungamente assaporati da Casanova. Nessun lettore potrebbe trovarvi un inizio, né una fine o un fine plausibili, né una logica, né un sistema, né uno sviluppo. A differenza di tanti suoi compagni di viaggio letterari da Gil Blas a Roderick Random - l'errante Casanova non cerca nulla, neppure una casa; giovanissimo abate a Martorano, fugge a Roma; soldato della Repubblica a Corfù, ritorna a Venezia nei panni di violinista; commissario del Lotto a Parigi, corre a Ferney; finalmente, bibliotecario a Dux del conte Waldstein, anche da Dux vorrebbe andarsene per sempre. Per poco che la si osservi, l'Histoire mostra la sua eccezionale "arte" di libro senz'argini, scritto contro l'idea stessa di libro e contro la forma, l'assertività, la conclusività del libro sull'inconcludibile vita.
\end{abstract}

La manipolazione del tempo somiglia in Casanova ad un'operazione necromantica fortemente connotata in senso ironico. Il tempo umano è irrevocabile nel suo decorso (la giovinezza non ritorna), ma un uso accorto degli strumenti retorici - orchestrando sapientemente il ritmo interno della diegesi - può fabbricare l'illusione di rivivere il passato in forma potenziata, assaporandone ogni stilla in una specie di immersione totalizzante e psicotropa, partecipe insieme del caos di un finto presente, continuamente in divenire, come della lucidità acuminata di uno sguardo sinottico, che tutto rivive e giudica a partire dal privilegio di una specie di prospettiva postuma. La conseguenza che sul piano architettonico se ne produce risulta duplice e ambivalente. Per un verso, essa porta a evocare il celebre

1 «La théorie des mœurs n'est d'autre utilité à la vie de l'homme que de celle qui résulte à celui qui avant de lire un livre en parcourt l'index: quand il l'a lu il ne se trouve informé que de la matière. Telle est l'école de morale que nous donnent les sermons, les précepts, et les histoires que nous content ceux qui nous élèvent. Nous écoutons tout avec attention; mais lorsque le cas nous arrive de mettre à profit les avis qu'on nous a donné, il nous vient envie de voir si la chose sera comme elle nous a été prédite: nous nous y livrons, et nous nous trouvons punis par le repentir. Ce qui nous dédommage un peu c'est que dans ces moments-là nous nous reconnaissons pour savants, et pour possesseurs du droit d'instruire les autres. Ceux que nous endoctrinons ne font ni plus ni moins de ce que nous avons fait, d'où il résulte que le monde reste toujours là, ou va de mal en pire» (CASANOVA, $2013: 59-60)$. 
paradosso - caro al Borges della Storia universale dell'infamia - di una mappa capace di descrivere l'impero in scala 1:1 (come una pellicola fedelmente sovrapposta al mondo). In Casanova una divorante febbre bulimica, uno spaventoso appetito di sempre nuova e risorgente vita trasforma l'autore dell' Histoire de ma vie in un logorroico narratore grafomane («la pensée taciturne ne me suffit pas; il faut que je parle», CASANOVA, 2013 : 518), maniacalmente invasato dall'urgenza di svolgere nei termini della massima evidenza il filo aggrovigliato di tutti i ricordi, e contemporaneamente protrarre il racconto all'infinito, dilazionandone l'impossibile conclusione per assaporare manieristicamente tutta l'ebbrezza implicita nell'atto gioioso di riavvolgere, attraverso la parola, il nastro del tempo: «Je ne sais pas si le lecteur sait ce que c'est qu'un amant qui prenant congé de l'objet qu'il aime craint de ne plus le revoir. Il fait le dernier compliment, et après l'avoir fait ne veut pas que ç'ait été le dernier, et il le renouvelle jusqu'à ce qu'il voie son âme distillée en sang» (CASANOVA, 2013 : 120). Da una parte, il referto tende insomma verso l'incomprimibile latitudine: preoccupato soprattutto di non concludere. Ma insieme, altrettanto forte e compresente, a governare i tempi della narrazione casanoviana si accampa la convinzione che «rien ne peut être plus cher à l'homme qui pense que la vie, et malgré cela le plus voluptueux est celui qui exerce le mieux l'art trop difficile de la faire passer vite. On ne veut pas la rendre plus courte; mais on veut que l'amusement rende son cours insensible» (CASANOVA, $2013: 769$ ).

Nella fabbricazione di questo utopico "tempo ritrovato", nessuno spazio è insomma concesso al vuoto della noia: in termini bergsoniani la simultaneità cavalca la durata, nello slancio di modellare "a tutto tondo" l'immagine vivida degli attimi presi in sequenza e incatenati, à bout de souffle. Per alimentare il prodigio di questo «ininterrotto andantino in cui il minimo avviso di dissonanza equivale alla più grave minaccia» (FICARA, 1999 : 89), Casanova non esita a forzare l'inerzia della realtà vile e prosaica, adulterando sagacemente il rapporto tra verità e menzogna (del resto: «la rhétorique n'emploie les secrets de la nature que comme les peintres qui veulent l'imiter. Tout ce qu'ils donnent de plus beau est faux», CASANOVA, $2013: 55)$. La relazione che l'Histoire de ma vie istituisce con il piano della verità fattuale - «riant en moi-même de toutes les fausses histoires, et des circonstances qui se combinent pour leur donner le caractère de la vérité» (CASANOVA, $2013: 284$ ) - può sempre ridursi a un semplice minuetto o una sorridente contraddanza, stagliata sul limite del flatus vocis e dell'assurdo. Spudorata ne appare la dichiarazione, per esempio, nel momento in cui Casanova inventa a Grenoble, per accreditarsi coi maggiorenti del luogo, una trascorsa amicizia tra il padre Gaetano e Jean-Pierre Bouchenu de Valbonnais (autore, nel 1722, di una Histoire du Dauphiné, al veneziano nota forse attraverso una pagina di Voltaire): «cette fable que j'ai inventée sur-le-champ parut un coup de théâtre, et me fit devenir une merveille. Nous nous levâmes tous à la fois, et nous allâmes recommencer le bal» (CASANOVA, 2015a : 464). 


\section{Una scrittura sonnambolica}

In questa simulazione volta a rendere utopicamente misurabile che «le temps qu'on emploie à la jouissance n'est jamais perdu» - per dirla con la celebre sentenza attribuita da CASANOVA (2015a : 882) alla deliziosa Marcoline -, il secondo espediente al quale il procedimento autobiografico dell' Histoire de ma vie copiosamente fa ricorso è l'equiparazione della realtà alla sottile allucinazione prodotta da un sonno vigile, correggendo in direzione esuberante e sensista («dans un songe, qui ne cédait en rien aux charmes de la réalité», CASANOVA, $2013: 519)$ il vecchio topos secentesco del mondo leggibile quale un teatro d'ombre. La meccanicità fredda e ponderata dell'operazione presenta tratti singolarmente proto-surrealisti: obbligandosi a sognare il proprio passato per riattingere i momenti perduti, il verbale delle memorie casanoviane produce una specie di delirante movimento en abîme, proiettando nel presente, in forma viva e pulsante, l'immagine di un passato nel quale l'esperienza prendeva corpo a partire da una cancellazione - essa stessa già operativa - dei confini tangibili fra la veglia e il sonno. Così avviene riassaporando - per fare solo un esempio - le sensazioni di un giovanile risveglio, dopo avere sognato di Henriette (CASANOVA, 2013 : 520):

Dans les rêves de cette espèce, ordinairement le rêveur se réveille un moment avant la crise. La nature, jalouse de la vérité, ne souffre pas que l'illusion aille tant en avant. Un homme qui dort n'est pas tout à fait vivant, et il doit l'être dans un instant dans lequel il peut donner à la vie un être semblable à lui-même. Mais, ô prodige! Je ne me suis pas réveillé, et j'ai passé toute la nuit avec Henriette entre mes bras. Mais quel long songe! Je n'ai pu le reconnaître pour songe que lorsque mon réveil à la pointe du jour l'a forcé à disparaitre. Je me suis tenu un bon quart d'heure immobile, et stupefait à en résumer les circonstances dans ma mémoire étonnée. Je me souvenais d'avoir plusieurs fois dit en dormant non je ne rêve pas: et j'aurais encore cru de n'avoir pas rêvé, si je n'avais pas trouvé la porte de ma chambre fermée au verrou par-dedans. Sans cela j'aurais cru qu'Henriette s'en fût allée avant que je me réveillasse, après avoir passé la nuit avec moi.

Inscritto nel cerchio del fondamentale ottimismo casanoviano (con la sua energica fiducia - di intonazione libertina - nella concreta misurabilità del mondo sotto specie fenomenica), il dissidio fra allucinazione e vita implica un finto problema gnoseologico, una forma ingannevole di esitante scetticismo, funzionale ad alimentare la trama del divertimento e dell'avventura dentro lo stravagante territorio del molteplice. La perdita del senso del reale è una specie di trompel'œil, le cui conseguenze si producono tutte nel registro imperturbabile di una comicità riscaldata al fuoco della geometrica intelligenza settecentesca. L'esito estremo può risultarne, al limite, la pantomima in sospetto di farneticazione (con le sorelle Marton e Nanette, per esempio, nei termini di un "gioco a tre" condizionato dalla reciproca consapevolezza di muoversi entro uno spazio vincolato a un obbligo di teatrale simulazione, quasi nei termini di una partita a scacchi): «J'ai d'abord fait semblant de dormir, mais un quart d'heure après, je me suis endormi tout de bon. Je ne me suis réveillé que quand elles vinrent se coucher; mais je me suis d'abord tourné pour reprendre mon sommeil, et je n'ai commencé à agir que quand je me suis vu le maître de les croire endormies. Si elles ne dormaient pas, il 
ne tenait qu'à elles d'en faire semblant. Elles m'avaient tourné le dos, et nous étions à l'obscur» (CASANOVA, 2013 : 100).

Fra allucinazione e danza, anche la rievocazione degli stati di annichilimento e paura - lievito decisivo, nel secolo dei Lumi, di ogni teorizzazione intorno al "sublime" - riesce modellata sulla medesima segreta istanza musicale. Disperato mentre rivisita il giovanile sconforto per avere dilapidato tutto (in una notte dissennata al tavolo verde di Chioggia), CASANOVA (2013 : 152) non rinuncia ad imprimere al proprio resoconto una specie di impercettibile accelerazione registica: «Ma nature accablée par ce grand malheur, eut besoin de s'y rendre insensible, se noyant dans le frère de la mort. Ce fut le sacré bourreau qui à midi me réveilla pour me dire d'un air triomphant qu'on avait invité à souper un jeune homme fort riche qui ne pouvait que perdre, et que par conséquent je me referais $[\ldots] \gg$. Con un sapiente gioco di specchi, il sonno posto a rimedio della catastrofe prodotta dall'iniziale rovescio partorisce un secondo orripilato disastro («l'espoir de regagner mon argent me fit perdre tout mon reste»), che Casanova rivive $a u$ ralenti scrivendo.

Nel movimento sinusoidale delle peripezie di fortuna, nessuna sventura è - per definizione - irrimediabile. Il piacere della scrittura implica in sé una enorme facoltà catartica. «La solitude sous les Plombs désespère - scrive CASANOVA (2013 : 908) -, mais pour le savoir il faut en avoir fait l'expérience. Si le prisonnier est un homme de lettres; qu'on lui donne un écritoire, et du papier, et son malheur diminue de neuf dixièmes». Scrivere è truccare le carte: è un gioco di prestigio per simulare il pieno davanti all'orrore del vuoto, del silenzio, dell'inazione. Nel resoconto casanoviano della prima carcerazione veneziana, la consapevolezza di questa necessaria commutazione si riversa in un incubo di trasparente cifra allegorica: aggrappato durante il sonno alla propria mano (nel vano desiderio di percepire una presenza umana dentro lo spazio angusto della cella), il prigioniero dei Piombi la avverte al risveglio come una parte separata del proprio corpo, minacciosamente vitale e cadaverica ad uno stesso tempo:

Rendu un peu à moi-même je me suis fait la grâce de croire que la main que j'avais cru de toucher n'était qu'un objet de l'imagination: dans cette ferme supposition j'allonge de nouveau le bras au même endroit, et je trouve la même main, que transi d'horreur, et jetant un cri perçant je serre, et je relâche en retirant mon bras. Je frémis; mais devenu maître de mon raisonnement, je décide que pendant que je dormais on avait mis près de moi un cadavre [...]. Cette aventure, quoique comique, ne m'a pas égayé. Elle m'a au contraire donné sujet aux réflexions les plus noires. Je me suis aperçu que j'étais dans un endroit, où si le faux paraissait vrai, les réalités devaient paraitre des songes; où l'entendement devait perdre la moitié de ses privilèges; où la fantaisie altérée devait rendre la raison victime ou de l'espérance chimérique, ou de l'affreux désespoir. (CASANOVA, $2013: 906)$

Da una prigione reale (i Piombi) ad una solo pallidamente metaforica (la biblioteca di Dux): importante, per Casanova, restava continuare a rendere possibile - fino all'ultimo - questa alchimia verbale romanzesca in cui «si le faux paraissait vrai, les réalités devaient paraître des songes», nell' autogoverno di una sorvegliata impostura. 


\section{La sincerità di Epimenide fingitore}

Alla metà del Settecento, nelle pagine di un romanzo dell'abate Pietro Chiari (La commediante in fortuna, 1755), la raffigurazione dell'avventuriero Casanova nei termini di un chiassoso e futile ciarlatano sembra già fissata precocemente alla stregua di un paradigma. Eppure l'immagine che questo esemplare imbonitore ama rivendicare per sé, in molti luoghi dell' Histoire de ma vie, risulta paradossalmente molto differente: il vezzo del memorialista Casanova è quello di rappresentarsi come un uomo che parla in genere con spudorata franchezza, coltivando l'esercizio orgoglioso e diretto della verità. Detto in altri termini: Casanova è un simulatore che dichiara apertamente la propria finzione, un attore dentro una macchina teatrale in cui nessuno crede fino in fondo a nessuno, perché tutti recitano nella consapevolezza della generale finzione.

«N'ayez point de confiance dans les paroles dorées qu'un homme plein de feu vous dira [...] - raccomanda il subdolo seduttore alla sedicenne Vesian -; gardezvous aussi de supposer des sentiments désintéressés dans ceux que vous verrez surpris par vos charmes: ils vous donneront de la fausse monnaie en quantité pour vous réduire à leur accorder la bonne. [...] Pour moi je suis sûr que je ne vous ferai pas de mal, et j'espère de vous faire du bien» (CASANOVA, 2013 : 1071). Nelle prime pagine dell' Histoire de ma vie, questa strategia che fa di Casanova, per certi aspetti, un ennesimo moderno emulo di Epimenide cretese, all'interno delle coordinate di un antico problema logico vecchio quanto l'Occidente (per così dire: dalle Confutazioni sofistiche di Aristotele fino ad un matematico del Novecento come Alfred Tarski), trova una formulazione teorica esplicita, quasi spudorata. «Il n'y a jamais eu au monde des sorciers; - scrive CASANOVA (2013:21) - mais leur pouvoir a toujours existé par rapport à ceux auxquels ils ont eu le talent de se faire croire tels». La simulazione, la capacità di simulare (e dissimulare) è - per il memorialista dell'Historie de ma vie - un progetto di potere. Un progetto che non ha nulla di mefistofelico, in quanto sta inscritto nell'ordine naturale (e morale) della vita. Indossare nuove maschere e uniformi produce semmai un divertimento narcisistico quasi sfrenato, simile alla voluttà che per Casanova comporta - in un lontano giorno del 1744, a Bologna - l'acquisto di una divisa da ufficiale: «Je n'ai jamais eu un plaisir de cette espèce pareil à celui que j'ai ressenti me voyant au miroir habillé ainsi. Je me trouvais fait pour être militaire, il me semblait d'être étonnant. Sûr de n'être connu de personne, je jouissais des histoires qu'on forgerait sur mon compte à mon apparition au café le plus fréquenté de la ville» (CASANOVA, $2013: 282$ ).

In nome di questo diritto insindacabile alla felicità, Casanova può simulare (e dissimulare) applicandosi a qualunque ruolo, fedele a quel motto stoico in cui era custodita l'unica bussola della sua condotta (sequere Deum: "obbedire alla sorte"). Se è vero che «l'homme a beaucoup plus de raison d'immoler tout à sa propre conservation que les souverains n'en ont pour conserver l'État» - come CASANOVA afferma (2013 : 978), per estinguere ogni sospetto di rimorso, davanti a certe conseguenze sanguinose di cui è disseminata la sua evasione dai Piombi -, in questa spregiudicata applicazione della ratio status a una "ragione degli individui" risiede anche la facoltà di cancellare ogni distinzione fra verità e 
simulazione. Il lato che resta in ombra, ai margini di una tale prospettiva, permane quello della sua sfacciata, quasi tracotante sincerità: rivendicata in termini che sembrano evocare la celebre chiusa del capitolo XXV del Principe (MACHIAVELLI, 1999: 385) sulla fortuna «amica de' giovani, perché sono meno respettivi, più feroci e con più audacia la comandano»:

C'est un bonheur constant que j'ai eu jusqu'à l'âge de cinquante ans quand je me suis trouvé dans l'oppression. D'abord que j'ai trouvé des honnêtes gens curieux de l'histoire du malheur qui m'accablait, et que je la leur contais, je leur ai toujours inspiré toute l'amitié qui m'était nécessaire pour me les rendre favorables et utiles. L'artifice que j'ai employé pour cela fut celui de conter la chose avec vérité sans omettre certaines circonstances qu'on ne peut dire sans avoir du courage. Secret unique que tous les hommes ne savent pas en usage; parce que la plus grande partie du genre humain est composée de poltrons. Je sais par expérience que la vérité est un talisman, dont les charmes sont immanquables pourvu qu'on ne la prodigue pas à des coquins. Je crois qu'un coupable, qui ose la dire à un juge intègre, est absous plus facilement qu'un innocent qui tergiverse. Bien entendu que le narrateur doit être jeune, ou pour le moins non vieux, car l'homme vieux a pour ennemi toute la nature. (CASANOVA, $2013: 129$ )

Giunto a sfiorare una specie di data-spartiacque, nel momento in cui (alla vigilia del cinquantesimo compleanno) Casanova ottiene di tornare a Venezia dopo l'antica fuga dai Piombi e la conclusione di una ventennale peregrinazione in contumacia, questo carattere improvvisamente ostile della natura divenuta arcigna dovette manifestarsi per lo scrittore-avventuriero esattamente con il volto della patria ritrovata. La Serenissima, nel concedergli il perdono sotto forma di amnistia, gli spalancava le porte di una quotidianità deprimente e grigia, senza prospettiva di gratificazioni o incarichi. È la ragione sostanziale per la quale Casanova - almeno nel progetto iniziale - si determina ad arrestare la propria autobiografia con la conclusione dell'anno 1774: come se il rientro a Venezia diventasse il sigillo di un irrimediabile addio alla giovinezza (precipitando, nell' affievolirsi di una sprezzante audacia, verso un futuro gravato solo di inerzia). Appoggiarsi a Machiavelli per denunciare la chiusura soffocante della vita politica veneziana potrebbe apparire esteriormente un paradosso: perché il senso comune del Settecento non ha mai dubbi nell'interpretare le pagine del Segretario fiorentino in chiave repubblicana, secondo una prospettiva che trova nel Diderot dell'Encyclopédie una «consacrazione» addirittura ufficiale (PROCACCI, 1995 : 294). Ma il quadro istituzionale della Repubblica di Venezia - congelato per secoli, dopo la Serrata del Maggior Consiglio (1297), in direzione severamente oligarchica - determina una specie di torva sclerotizzazione degli ordinamenti civili che proprio la lettura del repubblicano Machiavelli aiuta Casanova a intendere nella giusta luce, permettendogli di «comprendre le despotisme de sa patrie: au "devoir de se conserver", le gouvernement vénitien devait en effet "sacrifier tout", au point que pour lui "les lois mêmes cess[ai]ent d'être inviolables"» (LUNA, 1998 : 405).

La diagnosi casanoviana, su questa implicita impossibilità di adattarsi alle costrizioni della vita veneziana, aveva del resto comportato - nel primo libro dell' Histoire de ma vie - una precoce sanzione: laddove nel governatore dell' isola greco-veneziana di Zante - Giovanni-Antonio Dolfin (incontrato dallo scrittore, a vent' anni, sulla rotta che lo condurrà verso Costantinopoli) - CASANOVA (2013 : 
293-94) sembra costruire una specie di affascinante autoritratto per interposta persona:

Très éloquent, très poli, beau joueur qui perdait toujours, aimé de toutes les femmes desquelles il voulait l'être, toujours intrépide, et égal dans les bonheurs, et dans le travers de la fortune. Il avait voyagé sans permission, et étant tombé par conséquent en disgrâce du gouvernement, il s'était mis au service militaire d'une puissance étrangère. [...] Cet homme charmant, généreux, et point riche, eut besoin de demander au Grand Conseil un gouvernement lucratif; et on l'a élu conseiller dans l'île du Xante; mais il y allait avec un tel train qu'il ne pouvait pas espérer d'y gagner. Ce noble Venitien Dolfin, tel que je viens de le décrire ne pouvait pas faire fortune à Venise. Un gouvernement aristocratique ne peut aspirer à la tranquillité qu'ayant pour base, et pour maxime fondamentale l'égalité entre les aristocrates. Or il est impossible de juger de l'égalité soit physique, soit morale autrement que par l'apparence, d'où il résulte que le citoyen qui ne veut pas être persécuté, s'il n'est pas fait comme les autres, ou pire, doit employer toute son étude pour le paraître. S'il a beaucoup de talent, il doit le cacher; s'il est ambitieux, il doit faire semblant de mépriser les honneurs: s'il veut obtenir, il ne doit rien demander: s'il a une jolie figure il doit la négliger: il doit se tenir mal, se mettre encore plus mal, sa parure ne doit avoir rien de recherché, il doit tourner en ridicule tout ce qui est étranger; faire mal la révérence, ne pas se piquer d'un grande politesse, ne faire pas grand cas des beaux-arts; cacher son bon goût s'il l'a fin; ne pas tenir un cuisinier étranger, il doit porter une perruque mal peignée, et être un peu malpropre. M. Dolfin Bucintoro n'ayant aucune de ses qualités ne pouvait donc pas faire fortune dans Venise sa patrie.

Venezia appare in sostanza, agli occhi di Casanova, un piccolo mondo conformista fondato su un patto nobiliare che congela l'assetto sociale e soffoca ogni personale ambizione. Peggio - per tragicomico paradosso - la città è il luogo nel quale la dissimulazione delle qualità individuali diventa necessaria per la conservazione di una simulazione di concordia civile. Nell'insofferenza di Casanova, nella nettezza del suo giudizio, non c'è solo una pulsione libertina e quasi anarchica a rompere questa gerarchia soffocante; in lui viene avanti, rivendicata in modo esplicito, una professione orgogliosa di libertà mentale riflessa nella gioia senile della scrittura:

D'abord que je respectais les lois il me semblait de pouvoir mépriser les préjugés. Je croyais de pouvoir vivre parfaitement libre dans un pays sujet à un gouvernement aristocratique. Je ne serais trompé quand même la Fortune m'aurait fait devenir membre du gouvernement. La République de Venise, connaissant que son premier devoir est celui de se conserver, se trouve elle-même esclave de l'impérieuse raison d'État. Elle doit dans l'occasion sacrifier tout à ce devoir vis-à-vis duquel les lois même cessent d'être inviolables. [...] Assez riche, pourvu par la nature d'un extérieur imposant, joueur déterminé, panier percé, grand parleur toujours tranchant, point modeste, intrépide, courant les jolies femmes, supplantant des rivaux, ne connaissant pour bonne que la compagnie qui m'amusait je ne pouvais être que haï. Étant prêt à payer de ma personne je me croyais tout permis, car l'abus qui me gênait me paraissait fait pour être brusqué. (CASANOVA, $2013: 411$ )

C'è un conformismo simulato, in Casanova; e c'è una libertà intellettuale dissimulata. Le due cose stanno insieme, strettamente legate. Per orgoglio, CASANOVA (2013 : 121) può anche scegliere per sé la maschera dell'ignorante: accettando di farsi relegare, lui adulto di favoleggiato ingegno, in mezzo a un gruppo di bambini di nove anni alle prese con i primi rudimenti del latino (per non piegarsi all'umiliazione di subire un esame inutile, prima di entrare ventenne in seminario). «Socialmente di per sé poco rispettabile - ha scritto BENZONI (1998 : 
173) -, ogni volta la società, il bel mondo o presunto tale, l'accettano nella misura in cui [Casanova] s'adegua all'idea che di lui circola»: il che si inscrive totalmente nelle coordinate di una posizione - quella «dell'avventuriero e del parvenu» (BACHTIN, 2000: 273) - fortemente carica di implicazioni per lo sviluppo dell'intero romanzo europeo a partire dalla sua genesi primo-settecentesca.

\section{Le maschere di un moralista}

Incline a trasformare la propria memoria letteraria in «una fonte inesauribile di citazioni, aneddoti, digressioni» (SPAGGIARI, 2015 : 83), Casanova intrattiene con l'universo della tradizione italiana un rapporto peraltro assai disinibito e pragmatico, fondato sulla sostanziale volontà di riprodurre nel tessuto di questo francese eteroglosso - analogamente a quanto vale per il Goldoni dei Mémoires il «processo vitale della lingua che si fa nella conversazione» (FolENA, 1983: 367). Nel mondo ridotto a spazio teatrale, al giovane Casanova può capitare persino di fraternizzare in strada con un pontefice romano, Benedetto XIV, che la spregiudicatezza del ventenne Giacomo trasforma subito in maschera benevola di simpatico borghese («après avoir bien ri de ce que je lui ai dit de l'évêque il me dit que sans me gêner à lui parler toscan, je devais lui parler vénitien, comme il me parlait bolognais», CASANOVA, $2013: 218$ ). Non serve pensare che Machiavelli sia uno degli autori posti all'Indice, dalla cui proibizione Casanova chiede al papa di essere dispensato («je lui ai demandé la permission de lire tous les livres défendus»): poiché nessuna dispensa in verità gli occorreva per fare di Machiavelli, di «quel suo dire originalissimo e sugoso» (ALFIERI, 1977 : 178), un'esperienza fondativa del proprio modo di leggere in chiave teatrale e drammaturgica le cose della politica e del mondo.

Chi non capisce le regole della finzione, non sa vivere (da suddito) e non sa governare (da sovrano). Per esempio, in CASANOVA (2013: 1105) è chiaro ed esplicito - espresso con brevità machiavelliana - il giudizio feroce sulle mancate qualità politiche dell'imperatore viennese Giuseppe II:

\footnotetext{
Pour ce qui regarde l'art de régner il ne le connaissait pas, car il n'avait aucune connaissance du cœur humain, il ne savait ni dissimuler, ni garder le secret, il laissait voir le plaisir qu'il avait à punir, et il n'avait pas appris à régler sa physionomie. Il négligea cet artifice au point que lorsqu'il voyait quelqu'un qu'il ne connaissait pas, il faisait une grimace qui le rendait très laid, dans le moment qu'il aurait pu se permettre de substituer à cette grimace odieuse une lorgnette, car la grimace paraissait dire qui est donc cette espèce?
}

La dissimulazione come esercizio dell'intelligenza applicato al corpo, alla fisiognomica, alla sfera delle emozioni riflesse nel gesto e nel volto. Casanova, lettore di Tacito e di Machiavelli, sapeva bene che cosa la dissimulazione comporta in un principe (in un tiranno): un gioco freddo dell'intelligenza applicato a un'istanza potenzialmente sadica (il tiranno, dissimulando, diventa crudele come il gatto quando gioca con il topo). Ma poi non rappresenta, questo necessario governo delle proprie reazioni, delle proprie smorfie, della propria maschera, l'unico codice di sopravvivenza per tutti, dentro la macchina infernale del mondo? Per citare Ezio RAIMONDI (1972 : 250): «nei ghiribizzi del Machiavelli 
l'invenzione comica non esplode solo d'un tratto come un gesto dialogico, ma si condensa e fermenta all'interno del parlato: è un'acutezza intellettuale che reintroduce nella gioia fisica della farsa [...] le ambivalenze e i travestimenti di un autentico metateatro». Casanova, quando un giorno deve vedersela a Roma con l'infido conte Manuzzi, sembra avere appreso perfettamente la lezione di Machiavelli (come politico e come uomo di penna):

Fort étonné que cet homme qui avait poussé la vengeance jusqu'a vouloir me faire assassiner parle de moi comme d'un ami intime je me suis déterminé à dissimuler pour voir où la chose irait aboutir. Je l'ai donc vu paraître sans lui donner aucun marque de mon juste ressentiment, et lorsque après les compliments de devoir qu'il fit à l'ambassadeur, il vint à moi en position de vouloir m'embrasser, je l'ai rencontré en ouvrant les bras, et je lui ai demandé des nouvelles de l'ambassadeur. Il parla beaucoup à table disant pour me faire honneur vingt mensonges sur tout ce que j'avais fait à Madrid, en se félicitant, je crois, de ce que mentant il me forçait à mentir, m'invitant ainsi à en faire autant pour lui. (CASANOVA, 2015b : 738)

«La fourberie est vice; mais la ruse honnête n'est autre chose que la prudence de l'esprit. C'est une vertu. Elle ressemble, il est vrai, à la friponnerie, mais il faut passer par là. Celui qui ne sait pas l'exercer est un sot. Cette prudence s'appelle en grec cerdaleophron. Cerda veut dire renard» (CASANOVA, 2013 : 175). Mescolato con il ragionamento aristotelico sulla contiguità di vizio e virtù (nel secondo libro dell'Etica Nicomachea), il discorso casanoviano assolutizza il «bisogna adunque essere volpe» di MACHIAVELLI (1999 : 298) nel diciottesimo del Principe. Posizione sottilmente utilitaristica, dietro la quale preme tuttavia una tensione molto diversa. Essa corrisponde al modo severo e accigliato, da splendido moralista, con il quale lo scrittore giudica altrove, nell' Historie de ma vie, il bene e il male: in termini intellettualistici pensando, un poco alla maniera di Socrate, che dietro ogni malvagio stia nascosto uno stupido, perché non esistono nel mondo veri cattivi, ma soltanto veri stupidi.

Il rovescio di questa posizione può essere paradossale - «je n'ai jamais su décider si cet abbé Grimani était bon parce qu'il était bête, ou si la bêtise était un défaut de sa bonté» (CASANOVA, $2013: 166$ ) -; ma resta illuminante il modo in cui per esempio - nel caso di un personaggio degno dell'antica penna di Boccaccio, il frate Steffano incontrato sulla strada di Roma -, CASANOVA guarda (con la crudeltà di un Sancho cervantino) alla volgarità grottesca del religioso, sconcio imbonitore di piccole meschinerie (2013: 166):

C'était un sot qui avait l'esprit d'Arlequin, et qui supposait ceux qui l'écoutaient encore plus sots. Dans sa bêtise cependant il était fin. Sa religion était singulière. Ne voulant pas être bigot, il était scandaleux; pour faire rire la compagnie il disait des cochonneries révoltantes. Il n'avait le moindre goût ni pour les femmes, ni pour toute autre espèce d'impudicité, et il prétendait qu'on dût prendre cela pour une vertu tandis que ce n'était qu'un défaut de tempérament. Tout dans ce genre-là lui semblait matière à faire rire: et quand il était un peu gris, il faisait aux convives maris, femmes, et filles des questions si lubriques qu'il les faisait rougir. Le butor ne faisait qu'en rire.

Governata dall'intelligenza, la simulazione è per Casanova un arabesco sublime. Altrimenti è materia novellistica, comica, nella fondamentale assurdità dell'esperienza umana. 


\section{BIBLIOGRAFIA}

ALFIERI Vittorio (1977), Vita, a cura di Giulio Cattaneo, Milano, Garzanti.

ANGLANI Bartolo (1997), Il mestiere della metafora. Giuseppe Baretti intellettuale e scrittore, Modena, Mucchi.

BACHTIN Michail (2000), Estetica e romanzo, a cura di Rossana Platone, Torino, Einaudi.

BENZONI Gino (1998), Di corsa per l'Europa (anche per vivere intensamente), in : AA.VV., Il mondo di Giacomo Casanova. Un veneziano in Europa: 17251798, Venezia, Marsilio, pp. 167-177.

CASAnOva Giacomo (1913), Correspondance avec Johannes Ferdinand Opiz, Leipzig, Wolff Verlag.

CASANOVA Giacomo (2013), Histoire de ma vie (I-III), édition établie sous la direction de Gérard Lahouati et Marie-Françoise Luna, avec la collaboration de Furio Luccichenti et Helmut Watzlawick, Paris, Gallimard.

CASANOVA Giacomo (2015a), Histoire de ma vie (IV-VI), édition établie sous la direction de Gérard Lahouati et Marie-Françoise Luna, avec la collaboration de Furio Luccichenti et Helmut Watzlawick, Paris, Gallimard.

CASANOva Giacomo (2015b), Histoire de ma vie (VII-IX), édition établie sous la direction de Gérard Lahouati et Marie-Francoise Luna, avec la collaboration de Furio Luccichenti, Alexandre Stroev et Helmut Watzlawick, Paris, Gallimard.

CROTTI Ilaria (2005), Wunderkammern. Il Novecento di Parise e Comisso, Venezia, Marsilio.

FICARA Giorgio (1999), Casanova e la malinconia, Torino, Einaudi.

FOLENA Gianfranco (1983), L'italiano in Europa. Esperienze linguistiche del Settecento, Torino, Einaudi.

LEJEUNE Philippe (2004²), L'autobiographie en France, Paris, Armand Colin.

LUNA Marie-Françoise (1998), Casanova mémorialiste, Paris, Honoré Champion.

MACHIAVElli Niccolò (1999), Opere. I: De principatibus, Discorsi sopra la prima deca di Tito Livio, a cura di Rinaldo Rinaldi, Torino, Utet.

POULET Georges (1967), Casanova et le temps, in : BRANCA Vittore (éd.), Sensibilità e razionalità nel Settecento, vol. II, Firenze, Sansoni, pp. 691-714.

Poulet Georges (1977), Entre moi et moi. Essais critiques sur la conscience de soi, Paris, José Corti.

PROCACCI Giuliano (1995), Machiavelli nella cultura politica dell'età moderna, Roma-Bari, Laterza.

RAIMONDI Ezio (1972), Politica e commedia. Dal Beroaldo al Machiavelli, Bologna, il Mulino.

Restif De LA BRETONNE Nicolas-Edme (1959), Monsieur Nicolas, ou le Cour humain dévoilé, Paris, J.-J. Pauvert.

RuOzZI Gino (2012), Quasi scherzando. Percorsi del Settecento letterario da Algarotti a Casanova, Roma, Carocci.

Sollers Philippe (1999), Il mirabile Casanova. Apoteosi di un libertino, Milano, il Saggiatore. 
Spaggiari William (2015), Casanova, Pauline, la seduzione delle lettere, in : ID., Geografie letterarie. Da Dante a Tabucchi, Milano, Led, pp. 83-94.

VALÉRY Paul (1957), Stendhal, in ID., Euvres, tome I, édition établie et annotée par Jean Hythier, Paris, Gallimard, pp. 566-571.

VAROTTI Carlo (1998), Gloria e ambizione politica nel Rinascimento. Da Petrarca a Machiavelli, Milano, Bruno Mondadori. 